\title{
Quantitative evaluation of viral interference among Egyptian isolates of highly pathogenic avian influenza viruses (H5N1 and H5N8) with the lentogenic and velogenic Newcastle disease virus genotype VII in specific pathogen-free embryonated chicken eggs model
}

\author{
Mohamed A. Soliman, Ahmed A. Nour and Ahmed M. Erfan \\ National Laboratory for Veterinary Quality Control on Poultry Production, Animal Health Research Institute, Agricultural \\ Research Center, Dokki, Giza 12618, Egypt. \\ Corresponding author: Mohamed A. Soliman, e-mail: mohahm@hotmail.com \\ Co-authors: AAN: drahmednour83@yahoo.com, AME: ahmed.erfan10000@gmail.com \\ Received: 30-07-2019, Accepted: 11-10-2019, Published online: 23-11-2019
}

doi: www.doi.org/10.14202/vetworld.2019.1833-1839 How to cite this article: Soliman MA, Nour AA, Erfan AM (2019) Quantitative evaluation of viral interference among Egyptian isolates of highly pathogenic avian influenza viruses (H5N1 and H5N8) with the lentogenic and velogenic Newcastle disease virus genotype VII in specific pathogen-free embryonated chicken eggs model, Veterinary World, 12(11): 1833-1839.

\begin{abstract}
Background and Aim: Mixed infections of the highly pathogenic avian influenza virus (HPAIV) and Newcastle disease virus (NDV) are considered the most distressing problem of the poultry industry. The problem arises due to the influence of a hidden virus on the replication of another suspected virus. Consequently, misdiagnosis of the real cause of disease may become a source of infection for other healthy stock by transmission and dissemination of the hidden virus. This study aimed to determine the impact of HPAIV and NDV on each other in a specific pathogen-free embryonated chicken egg (SPF-ECE) model.

Materials and Methods: HPAIVs (H5N1 and H5N8) and NDVs [avirulent NDV [avNDV] and velogenic NDV [vNDV]) were inoculated into the allantois cavity of SPF-ECE with graded titers $\left(2,3\right.$, and $4 \log _{10}$ EID50) at 24 and $48 \mathrm{~h}$ of incubation, followed by the collection of allantoic fluid. A quantitative reverse transcription real-time polymerase chain reaction was used to determine the viral RNA copies of both viruses.

Results: Obvious interference was reported on the growth of NDVs when co-inoculated with AIVs. NDV RNA titers reduction ranged from $<3$ to $5 \log _{10}$ to complete suppression, but slight interference with the growth of AIVs occurred. H5N1 RNA titers showed $<1-2 \log _{10}$ reduction when co-inoculated with vNDV compared with the H5N1 control. The interference impact of H5N8 was more powerful than that of H5N1, while vNDV showed more resistance for interference than the avNDV strain. On the other hand, interference of AIVs was not observed except when vNDV was inoculated before H5N1. The interfering impact was increased after $48 \mathrm{~h}$ of inoculation, whereas no titer of avNDV was detectable.
\end{abstract}

Conclusion: AIV strains had a powerful effect on NDV growth, regardless of which infection occurred first.

Keywords: avian influenza virus, Newcastle disease virus, real-time polymerase chain reaction, viral interference.

\section{Introduction}

Avian influenza (AI) and Newcastle disease (ND) are two major viral diseases that cause major losses to the poultry industry [1]. During the past decade, the poultry industry in Egypt was overwhelmed by the exposure to different AI virus (AIV) subtypes including the low pathogenic AIV (LPAIV) AI H9N2 and highly pathogenic AIVs (HPAIV) (HPAIV H5N1 and HPAIV H5N8) [2-4]. Meanwhile, ND continues to cause serious problems and high economic losses in the Egyptian poultry industry [5]. The genetic evolution of HPAIV in Egypt has been suggested to produce new clades 2.2.1.2 H5N1 and 2.3.4.4 H5N8; this raises the question of the effect

Copyright: Soliman, et al. Open Access. This article is distributed under the terms of the Creative Commons Attribution 4.0 International License (http://creativecommons.org/licenses/ by/4.0/), which permits unrestricted use, distribution, and reproduction in any medium, provided you give appropriate credit to the original author(s) and the source, provide a link to the Creative Commons license, and indicate if changes were made. The Creative Commons Public Domain Dedication waiver (http:// creativecommons.org/publicdomain/zero/1.0/) applies to the data made available in this article, unless otherwise stated. of coinfection with other endemic viruses [6]. Mixed infection of both viruses caused major problems for the poultry industry due to severe economic losses and the wide range of infection that is accompanied by high morbidity and mortality as well as decreased egg production $[7,8]$. Several studies provide evidence for the high incidence of NDV-AIV mixed infections [8-10]. The prior growth of NDV may inhibit AIV growth resulting in false-negative AIV tests [11]. In a coinfection study, LPAIV had a negative impact on NDV growth when they were inoculated simultaneously or sequentially [12]. The previous infection of specific pathogen-free (SPF) chickens with virulent NDV strains can suppress HPAIV as a result of competition for cell surface receptors or competent cells required for replication [13]. The pre-infection of a host with one virus may affect the multiplication of a second virus, a phenomenon known as viral interference [14]. Veterinary authorities and poultry producers face the problem of mixed infections which are complicated by false diagnosis, the effect of one virus on another, and serious viral dissemination or 
a source of transmission [15]. Some research used chicken embryos as a model for studying mixed infection of AIV and NDV and their interference [16], where clinical and serological parameters were the predominant tools for studying the interference of mixed viral infection for poultry. Though, studies that quantitatively evaluate the degree of interference between both viruses are lacking [17]. On the other hand, studies on interference between AIV and NDV showed variable conclusions [15].

So, the significance of the current study become maximized as it discussed viral interference by evaluating AIV and NDV viral replication using Quantitative reverse transcription real-time polymerase chain reaction (qrRT-PCR).

This study aimed to evaluate the impact of viral interference by the dual infection of AIVs (H5N1H5N8) and NDVs (avirulent NDV [avNDV]-velogenic NDV [vNDV]) in an SPF-embryonated chicken egg (SPF-ECE) model system using qrRT-PCR.

\section{Materials and Methods \\ Ethical approval}

This study does not require ethical approval as study was based on SPF-egg model (not living bird model).

\section{Virus strains}

Four standard titrated viruses (of $10^{6}$ EID50 titer) were obtained from the repository of the National Laboratory for Veterinary Quality Control on Poultry Production (NLQP), Egypt [HPAIVH5N1 (A/chicken/Egypt/173CAL/2017; HPAIV H5N8 (A/chicken/Egypt/CA35/2017; vNDV (NDVGHB-328F-2016); and avNDV (NDV-CH-BehairaEgypt-MR6-2012)]. GenBank accessions (for hemagglutinin [HA] gene for $\mathrm{H} 5 \mathrm{~N} 1$ and $\mathrm{H} 5 \mathrm{~N} 8$ AIVs and $\mathrm{F}$ gene for vNDV and avNDV NDVs) of the obtained strains are MG192004; MH762131; KX686728; and JX193771, respectively. Virus strains were 10-fold serially diluted to get the applied inoculum concentrations $\left(10^{2}, 10^{3}\right.$, and $10^{4}$ EID50). The viral infectivity of each strain was determined by serial titration in 10-11-days-old embryonated eggs and was expressed as $50 \%$ of the egg infective dose (EID50/mL) using standard methods [18].

\section{SPF-ECE inoculation}

SPF-ECEs were purchased from the Egyptian SPF Egg Production Farm (Nile SPF), Kom Oshiem, El-Fayoum Governorate, Egypt. ECEs were inoculated through the allantoic sac according to the OIE guidelines [19] and eggs were incubated at $37^{\circ} \mathrm{C}$. Inoculated eggs were candled daily for 3-5 successive days. Bacteria-free allantoic fluid was aliquoted and stored at $-80^{\circ} \mathrm{C}$ until tested.

\section{qrRT-PCR}

Viral RNA extraction from the harvested allantoic fluids was performed using QIAamp viral RNA Mini kit (Qiagen, GmbH, Germany). The AIV-H5 HA gene and NDV matrix $(\mathrm{M})$ gene were titrated in the purified RNA using standard QuantiNova real-time PCR kit (Qiagen, GmbH, Germany). qrRT-PCR was performed on a Mx3005P QPCR System (Agilent, California, USA). Samples with a $\mathrm{Cq}$ value $\leq 39$ were considered positive. QrRT-PCR primers and probes for AIV-H5 gene [20] and NDV matrix gene [21] were supplied by Metabion (Germany).

\section{Experimental designs for reciprocal interference studies between AIVs and NDVs}

The interference phenomenon was studied by two experiments, described below:

\section{Experiment 1}

Ten-days-old SPF-ECEs were sequentially infected with two viruses at equal multiplicities. In Table-1, a summary of the experimental design was provided. Four sets (sets 1-4) were divided individually into two groups (G1 and G2) of 15 eggs per group, each group was subdivided into three subgroups of varied titers 2, 3, and $4 \log 10$ (five eggs per titer). The first set was designed for sequential inoculation of G1: Inoculation of AIV H5N1 followed by avNDV and G2: Inoculation of avNDV followed by H5N1 with $12 \mathrm{~h}$ in between the two inoculations. The set 2 design was the same as set 1 , but with vNDV inoculation instead of avNDV. Sets 3 and 4 designs were the same as sets 1 and 2 designs, respectively (with inoculation of H5N8 instead of H5N1). Four positive control groups were designed for a single inoculation of each viral type, while sterile phosphate-buffered saline was injected instead of the second inoculum. One negative control group was also designed. After viral inoculations, ECEs were candled at $12 \mathrm{~h}$ intervals. Collection of allantoic fluid was done after $24 \mathrm{~h}$.

\section{Experiment 2}

The same protocol was performed as experiment 1, but the collection of allantoic fluid was performed after $48 \mathrm{~h}$. All the experiments were performed in duplicates.

\section{Statistical analysis}

RNA copy titer of each virus was determined using qrRT-PCR. The degree of interference was estimated by comparing AIV or NDV yields from coinfected ECEs with those of the corresponding controls as measured independently by qrRT-PCR. Statistical variation between the experimental group and control group was determined by ANOVA test where $p<0.05$. This experiment was set up to investigate the effect of the first inoculated virus on the growth of a second inoculated virus in the ECE.

\section{Results}

The results of experiments $(1,2)$ are shown in Figure-1, which represent the quantitative measure of H5N1 with reciprocal infection of avNDV and vNDV. AIV (H5N1) titers showed no reduction when ECEs were coinfected with avNDV. However, H5N1 RNA 


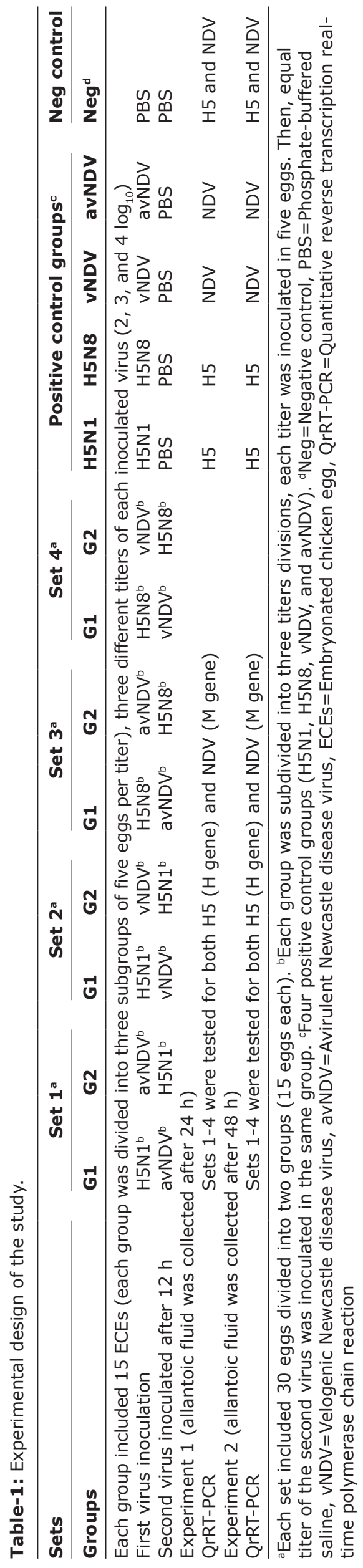

titers showed $<1-2 \log _{10}$ reduction when co-inoculated with vNDV compared with H5N1 control.

AIV (H5N8) titers showed no reduction when ECEs were coinfected with avNDV (Figure-2). However, H5N8 RNA titers showed one $\log _{10}$ reduction when co-inoculated with vNDV. The replication (irrespective of pre- or post-NDV infection) was compared to control single infection in ECE.

Virus yield of vNDV from dually infected ECE with $\mathrm{H} 5 \mathrm{~N} 1$ and $\mathrm{H} 5 \mathrm{~N} 8$ was significantly lower than those from singly infected ECE by a range of $<3-5 \log _{10}$ to complete inhibition (Figure-3a and $b$ ). The higher the level of infection with AIV virus, the greater the degree of interference observed. However, a prominent difference was found among $\mathrm{H} 5 \mathrm{~N} 1$ or $\mathrm{H} 5 \mathrm{~N} 8$ and virulent strain of NDV, where virulent NDV was more powerful in resisting interference induced by AIV (H5N1) (Figure-3a and b). Furthermore, findings showed that $\mathrm{H} 5 \mathrm{~N} 8$ was more powerful than $\mathrm{H} 5 \mathrm{~N} 1$ in inhibiting vNDV.

When equal titers of AIVs (H5N1 or H5N8) were sequentially inoculated, indications of interference on avNDV were observed (Figure-4). Only a high concentration of avNDV can slightly resist interference by H5N1 $(p<0.05)$. No significant difference in reciprocal inoculation of NDV with AIV (H5N1 or H5N8) was recorded. The avNDV multiplicity interfered irrespective of which virus was inoculated first. It was obvious that the high titer of avNDV $\left(4 \log _{10}\right)$ in infected ECE was relatively resistant to interference (Figure-4a). Another finding was that $\mathrm{H} 5 \mathrm{~N} 8$ was a more powerful agent than $\mathrm{H} 5 \mathrm{~N} 1$ in preventing the replication of NDV. As no viral yield of avNDV in different concentrations was detected (Figure-4b), whereas after $24 \mathrm{~h}$ post-inoculation of 3 and $4 \log _{10} \mathrm{H} 5 \mathrm{~N} 1$, a relative interference of avNDV was observed. However, after $48 \mathrm{~h}$ post-inoculation, complete interference of avNDV was recorded.

In view of the interfering effect of NDV (avNDV and VNDV) on AIV, NDV failed to inhibit the growth of AIV. The interference phenomenon was different after $48 \mathrm{~h}$ post-inoculation. The highest growth inhibition of NDV was observed when H5N8 was inoculated earlier than avNDV (Figure-4). Relative inhibition of vNDV with H5N8 and H5N1 was observed.

\section{Discussion}

Viral interference is a common episode where one cell is infected with a virus that can inhibit the replication of secondary homologous or heterologous viruses [14]. In this study, interference of $\mathrm{H} 5 \mathrm{~N} 1$ and H5N8 HPAIV AIVs with avNDV and vNDV was quantitatively evaluated in ECE model using qrRTPCR. Similar studies have shown that one virus is capable of inhibiting the growth of another [22,23]. The current study focused on the evaluation of the interference between AIV and NDV using qrRT-PCR to determine the degree of viral replication. Further, we studied the effect of some factors such as interfering doses, interfering intervals, and strain virulence. 


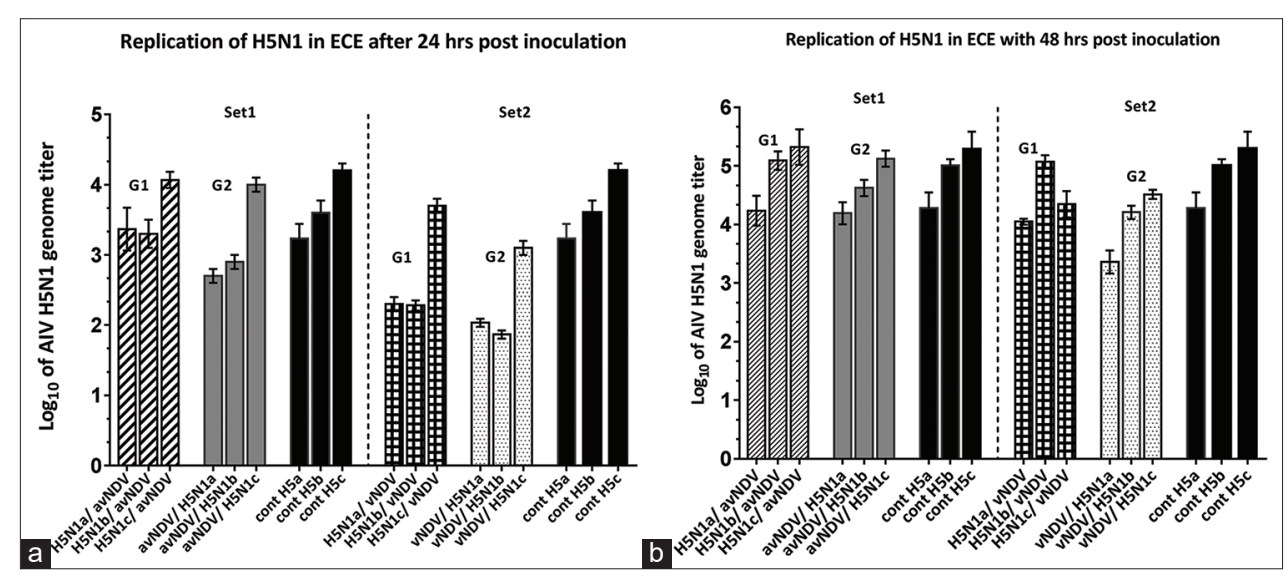

Figure-1: ( $a$ and $b)$ The replication titers of H5N1 in sets 1 and 2. Set 1 [G1 (H5N1/avNDV), G2 (avNDV/H5N1)]. Set 2 [G1 (H5N1/vNDV), G2 (vNDV/H5N1)]. Black columns represent the positive control group (single H5N1 inoculation). a-c represent three different inoculated titers $\left(2,3\right.$, and $4 \log _{10}$, respectively). Bars over the columns represent the error bars of standard deviation to the mean titers. $\mathrm{p}<0.05$. a is related to $24 \mathrm{~h}$ incubation, $\mathrm{b}$ is related to $48 \mathrm{~h}$ incubation.

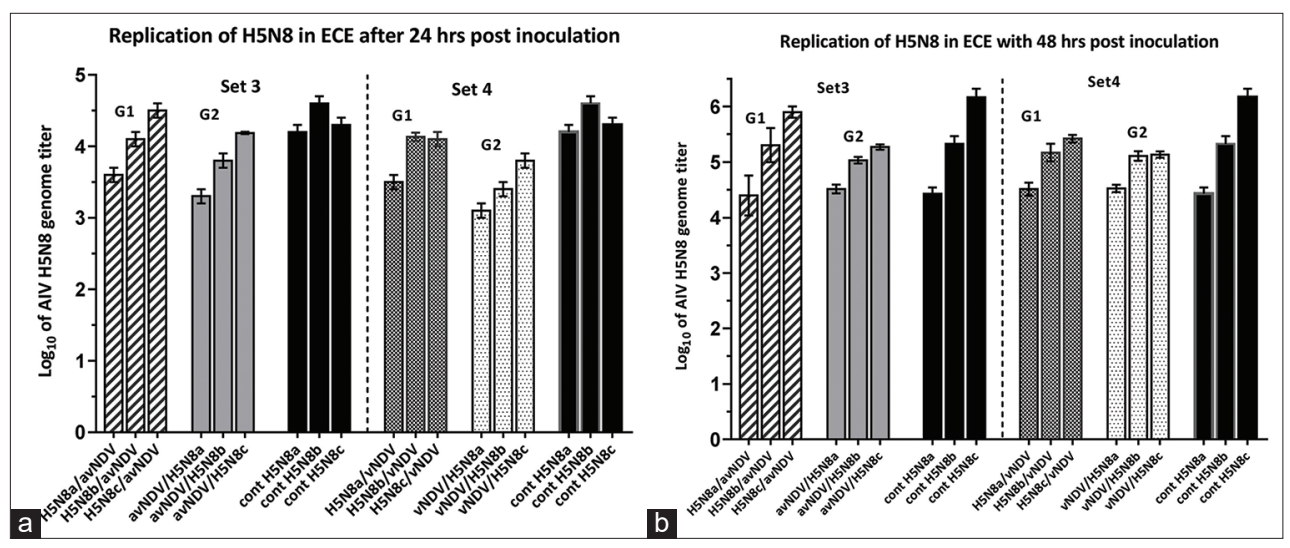

Figure-2: ( $a$ and b) The replication titers of H5N8 in sets 3 and 4. Set 3 [G1 (H5N8/avNDV), G2 (avNDV/H5N8)]. Set 4 [G1 (H5N8/vNDV), G2 (vNDV/H5N8)]. Black columns represent the positive control group (single H5N8 inoculation). a-c represent three different inoculated titers $\left(2,3\right.$, and $4 \log _{10}$, respectively). Bars over the columns represent the error bars of standard deviation to the mean titers. $\mathrm{p}<0.05$. a is related to $24 \mathrm{~h}$ incubation, $\mathrm{b}$ is related to $48 \mathrm{~h}$ incubation.

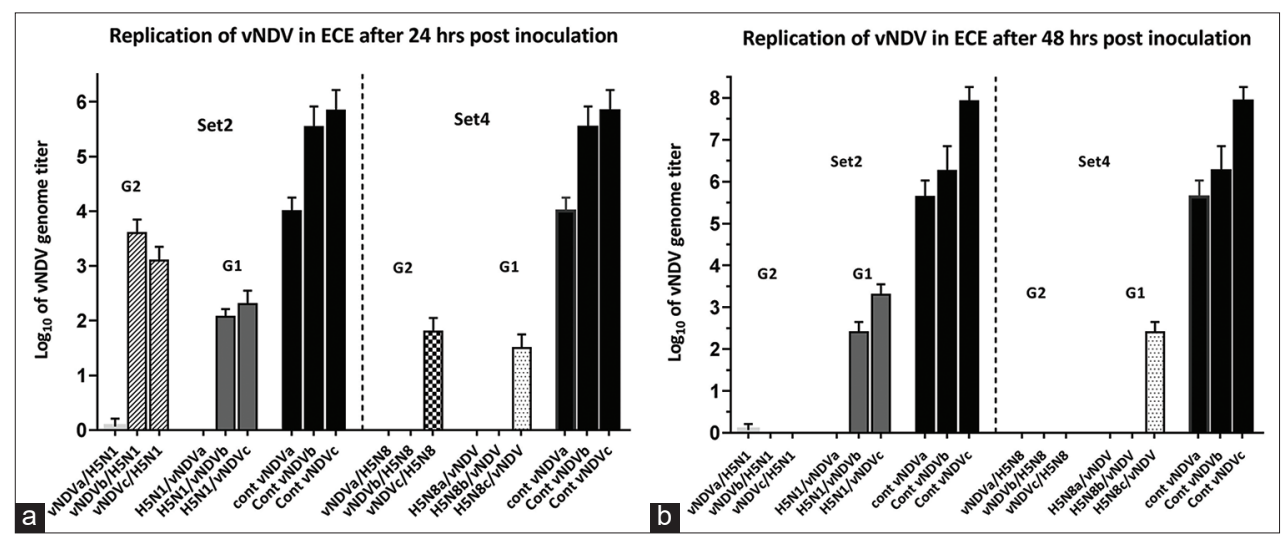

Figure-3: ( $a$ and $b$ ) The replication titers of vNDV in sets 2 and 4. Set 2 [G2 (VNDV/H5N1), G1 (H5N1/VNDV)]. Set 4 [G2 (vNDV/H5N8), G1 (H5N8/vNDV)]. Black columns represent the positive control group (single vNDV inoculation). a-c represent three different inoculated titers $\left(2,3\right.$, and $4 \log _{10}$, respectively). Bars over the columns represent the error bars of standard deviation to the mean titers. $\mathrm{p}<0.05$. a is related to $24 \mathrm{~h}$ incubation, $\mathrm{b}$ is related to $48 \mathrm{~h}$ incubation.

AIV inhibited the growth of NDV, and the observed interference grade ranged from partial to complete interference according to titer and virulence of the viral strains. NDV downregulated AIV replication by one log when NDV was inoculated 24 or $48 \mathrm{~h}$ before AIV, allowing for an increase in replication. In contrast to a related study [17], the primary infection by
NDV succeeded to inhibit to a lesser extent $\left(<1-2 \log _{10}\right)$ the later AIV replication (Figures-1 and 2).

However, our result was corroborated by the previous research that supported the concept that pre-infection with NDV (either lentogenic or velogenic) can minimize later LPAIV or HPAIV AIVs replications [13]. 

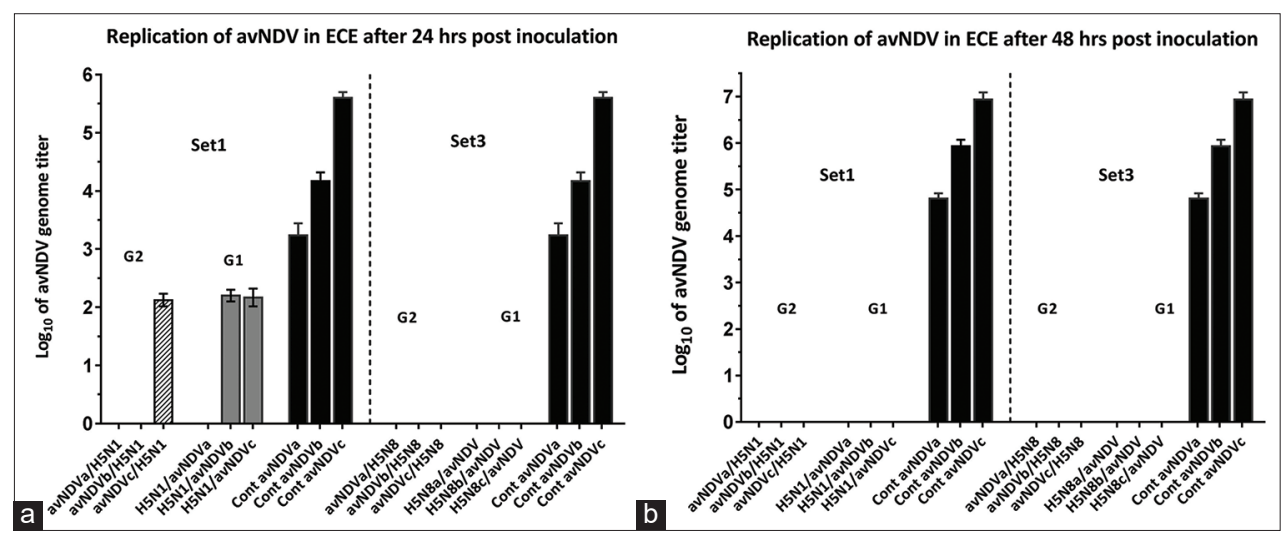

Figure-4: ( $a$ and b) The replication titers of avNDV in sets 1 and 3. Set 1 [G2 (avNDV/H5N1), G1 (H5N1/avNDV)]. Set 3 [G2 (avNDV/H5N8), G1 (H5N8/avNDV)]. Black columns represent the positive control group (single avNDV inoculation). a-c represent three different inoculated titers $\left(2,3\right.$, and $4 \log _{10}$, respectively). Bars over the columns represent the error bars of standard deviation to the mean titers. $\mathrm{p}<0.05$. a is related to $24 \mathrm{~h}$ incubation, $\mathrm{b}$ is related to $48 \mathrm{~h}$ incubation.

In contrast, in the varied path of this study, the first inoculation of $\mathrm{H} 5 \mathrm{~N} 8$ could inhibit the replication of subsequent avNDV or vNDV inoculums for up to $<2-3 \log _{10}$ concentrations, but vNDV at high concentration like $4 \log _{10}$ showed partial interference (Figure-3). This finding came in accordance with a study that reported that the previous infection of chickens with vNDV strains can reduce HPAIV replication. This interference depends on the viral titer, the virulence of NDV, and the timing of infections [24]. Furthermore, H5N1 could suppress the replication with vNDV or avNDV at $2 \log _{10}$, but partial interference with high concentration 3 or $4 \log _{10}$ was recorded only with vNDV though complete suppression was exerted by $\mathrm{H} 5 \mathrm{~N} 1$ on avNDV replication (Figures-3 and 4). Both outcomes suggest that vNDV is more potent in withstanding AIV interference than avNDV. In addition, the virulence of the viral strains was another important factor that affects interference in the current study. A previous study [25] reported the direct correlation between NDV strain virulence and the degree of replication.

A preceding study [26] also reported AIV-H9 interference due to NDV replication in ECEs. Another study was performed in SPF chickens that indicated that the previous infection of NDV can decrease the replication of HPAIV of H5N2 subtype [24]. A similar but more powerful interfering effect was reported by the previous study [11] where LPAIV-H9 was completely undetectable in cloacal swabs after Lasota vaccination.

Other studies reported the interfering effect exhibited by LPAIV-H7N2, where it significantly decreased the oral shedding of NDV in turkeys and chickens [13]. A resembling interfering effect was reported in a recent study where LPAIV-H9N2 delayed and decreased NDV shedding. They showed that the degree of viral interference is dose-dependent, which came in accordance with the findings in this study that the higher AIV inoculum dose reveals greater interfering effects on NDV replication [27].

Viruses exert their interfering action either through competing for cellular attachment as they reduce or even block the free cell receptors or by competing intracellularly for replication machinery [28].

The more probable AIV-NDV interference mechanism is the competition for cell receptor attachment as both viruses require sialic acid receptors either in the form of sialic acid-containing glycol conjugates for AIV [29] or gangliosides and N-glycoproteins for NDV [30].

Another mechanism for viral interference may be due to interferon induction due to primary viral infection that can suppress the replication of the secondary virus [31]. Such mechanisms were shown to be a possible mechanism for AIV-NDV interference [23]. This mechanism elucidates the strong inhibition of avNDV even when it was the primary infectious virus, as lentogenic NDV is a weak interferon inducer. The previous studies directly correlate the degree of interferon induction with the time interval between two infecting viruses [17,31]. This was taken into consideration in the current study, as there was a $12 \mathrm{~h}$ lag between the two viral inoculums to allow for maximum interferon activation. The current findings agree with the interference pattern exerted by interferon induction.

In the current study, we also report that $\mathrm{H} 5 \mathrm{~N} 8$ had a more powerful interfering force than H5N1. This complicates the case since in 2017, H5N8 HPAIV was in circulation in all Egyptian poultry sectors more than $\mathrm{H} 5 \mathrm{~N} 1$, so the chance of interference with NDV is currently higher than in the years before the entry of H5N8 in Egypt.

Although the interfering impact of avNDV was not obvious, it may lead to the misdiagnosis of AIV in coinfections with the Lasota strain due to lowering viral titers to an undetectable level that confound the correct diagnosis [10].

The study clarified the stronger capacity of vNDV compared with avNDV, in resisting the inhibition of AIV (inoculated later), also clarified the greater interfering capacity of $\mathrm{H} 5 \mathrm{~N} 8$ over that of H5N1, which was clearly demonstrated in this study. 


\section{Conclusion}

We summarize that AIV-NDV viral interference exists with a higher chance for AIV to inhibit NDV replication; however, the degree of interference may differ according to viral concentrations and strain virulence. Such episodes should be taken into consideration during field cases diagnosis to avoid false-negative results. The current study provides evidence that testing of interfering viruses during the molecular screening and viral isolation attempts of infected poultry flocks should be performed to identify the consequences of interference during coinfection. Finally, this study sheds light on the importance of planning for the diagnosis and control of avian disease among poultry sectors.

\section{Recommendations}

The current search recommends studying the viral interference between AIVs and NDVs in living bird model.

\section{Authors' Contributions}

MAS designed this study and applied statistical analysis. AME performed molecular biology tests. AAN performed viral inoculations. All authors drafted, revised the manuscript, analyzed the data, and approved the final manuscript.

\section{Acknowledgments}

The authors thank National Laboratory for Veterinary Quality Control on Poultry Production, Animal Health Research Institute, Egypt for funding this work (Grant No. NLQP-052018).

\section{Competing Interests} interests.

The authors declare that they have no competing

\section{Publisher's Note}

Veterinary World remains neutral with regard to jurisdictional claims in published institutional affiliation.

\section{References}

1. Capua, I. and Alexander, D.J. (2009) Avian influenza and Newcastle Disease: A Field and Laboratory Manual. Springer Verlag, Italy.

2. El-Zoghby, E.F., Arafa, A.S., Hassan, M.K., Aly, M.M., Selim, A., Kilany, W.H., Selim, U., Nasef, S., Aggor, M.G., Abdelwhab, E.M. and Hafez, H.M. (2012) Isolation of H9N2 avian influenza virus from bobwhite quail (Colinus virginianus) in Egypt. Arch. Virol., 157(6): 1167-1172.

3. Kayali, G., Kandeil, A., El-Shesheny, R., Kayed, A.S., Maatouq, A.M., Cai, Z., McKenzie, P.P., Webby, R.J., El Refaey, S., Kandeel, A. and Ali, M.A. (2016) Avian influenza A (H5N1) virus in Egypt. Emerg. Infect. Dis., 22(3): 379-388.

4. Selim, A.A., Erfan, A.M., Hagag, N., Zanaty, A., Samir, A.H., Samy, M., Abdelhalim, A., Arafa, A.A., Soliman, M.A., Shaheen, M., Ibraheem, E.M., Mahrous, I., Hassan, M.K. and Naguib, M.M. (2017) Highly pathogenic avian influenza virus (H5N8) Clade 2.3.4.4 infection in migratory birds, Egypt. Emerg. Infect. Dis., 23(6): 1048-1051.

5. Saad, A.M., Samy, A., Soliman, M.A., Arafa, A., Zanaty, A.,
Hassan, M.K., Sultan, A.H., Bazid, A.I. and Hussein, A.H. (2017) Genotypic and pathogenic characterization of genotype VII Newcastle disease viruses isolated from commercial farms in Egypt and evaluation of heterologous antibody responses. Arch. Virol., 162(7): 1985-1994.

6. Arafa, A., El-Masry, I., Kholosy, S., Hassan, M.K., Dauphin, G., Lubroth, J. and Makonnen, Y.J. (2016) Phylodynamics of avian influenza clade 2.2.1 H5N1 viruses in Egypt. Virol. J., 13(49): 1-11.

7. Jahangir, A., Ruenphet, S., Ueda, S., Ueno, Y., Shoham, D., Shindo, J., Okamura, M., Nakamura, M. and Takehara, K. (2009) Avian influenza and Newcastle disease viruses from Northern Pintail in Japan: Isolation, characterization and inter-annual comparisons during 2006-2008. Virus Res., 143(1): 44-52.

8. Hassan, K.E., Shany, S.A., Ali, A., Dahshan, A.H., El-Sawah, A.A. and El-Kady, M.F. (2016) Prevalence of avian respiratory viruses in broiler flocks in Egypt. Poult. Sci., 95(6): 1271-1280.

9. Amer, M.M., Maatouq, A.M., Abdel-Alim, G.A., Awaad, M.H.H. and Kutkat, M.A. (2018) Isolation and Identification of H9N2 avian influenza and Newcastle disease viruses co-infections in chicken Egypt. J. Vet. Sci., 49(2): 135-146.

10. Ellakany, H.F., Gado, A.R., Elbestawy, A.R., Abd El-Hamid, H.S., Hafez, H.M., Abd El-Hack, M.E., Swelum, A.A., Al-Owaimer, A. and Saadeldin, I.M. (2018) Interaction between avian influenza subtype H9N2 and Newcastle disease virus vaccine strain (LaSota) in chickens. BMC. Vet. Res., 14(1): 358.

11. El Zowalaty, M.E., Chander, Y., Redig, P.T., Abd El Latif, H.K., El Sayed, M.A. and Goyal, S.M. (2011) Selective isolation of avian influenza virus (AIV) from cloacal samples containing AIV and Newcastle disease virus. $J$. Vet. Diagn. Invest., 23(2): 330-332.

12. Fazel, P. and Mehrabanpour, M.J. (2018) Evaluation of the viral interference between lentogenic Newcastle disease virus (LaSota) and avian influenza virus (H9N2) using realtime reverse transcription polymerase chain reaction in SPF chicken. Braz. J. Poult. Sci., 20(3): 537-546.

13. Costa-Hurtado, M., Afonso, C.L., Miller, P.J., Spackman, E., Kapczynski, D.R., Swayne, D.E., Shepherd, E., Smith, D., Zsak, A. and Pantin-Jackwood, M. (2014) Virus interference between H7N2 low pathogenic avian influenza virus and lentogenic Newcastle disease virus in experimental co-infections in chickens and Turkeys. Vet. Res., 45(1): 1.

14. Kimura, Y., Norrby, E., Nagata, I., Ito, Y. and Shimokata, K. (1976) Homologous interference induced by a temperature-sensitive mutant derived from an HVJ (Sendai virus) carrier culture. J. Gen. Virol., 33(2): 333-343.

15. Umar, S., Guerin, J.L. and Ducatez, M.F. (2016) Low pathogenic avian influenza and coinfecting pathogens: A review of experimental infections in avian models. Avian Dis., 61(1): 3-15.

16. Shortridge, K.F. and King, A.P. (1983) Cocultivation of avian orthomyxoviruses and Para-myxoviruses in embryonated eggs: Implications for surveillance studies. Appl. Environ. Microbiol., 45(2): 463-467.

17. Ge, S., Zheng, D., Zhao, Y., Liu, H., Liu, W., Sun, Q., Li, J., Yu, S., Zuo, Y., Han, X., Li, L., Lv, Y., Wang, Y., Liu, X. and Wang, Z. (2012) Evaluating viral interference between Influenza virus and Newcastle disease virus using real-time reverse transcription polymerase chain reaction in chicken eggs. Virol. J., 9(128): 1-8.

18. Reed, L.J. and Muench, H. (1938) A simple method of estimating fifty percent endpoints. Am. J. Epidemiol., 27(3): 493-497.

19. OIE-World Organization for Animal Health. (2012) Manual of Diagnostic Test and Vaccines for Terrestrial Animals: Mammals, Birds and Bees. OIE-World Organization for Animal Health, Paris.

20. Löndt, B.Z., Nunez, N., Banks, J., Nili, H., Johnson, L.K. and Alexander, D.J. (2008) Pathogenesis of highly 
pathogenic avian influenza A/turkey/Turkey/1/2005 H5N1 in Pekin ducks (Anas platyrhynchos) infected experimentally. Avian Pathol., 37(6): 619-627.

21. Wise, M.G., Suarez, D.L., Seal, B.S., Pedersen, J.C., Senne, D.A., King, D.J., Kapc-zynski, D.R. and Spackman, E.E. (2004) Development of a real-time reverse-transcription PCR for detection of Newcastle disease virus RNA in clinical samples. J. Clin. Microbiol., 42(1): 329-338.

22. Roussan, D.A., Haddad, R. and Khawaldeh, G. (2008) Molecular survey of avian respiratory pathogens in commercial broiler chicken flocks with respiratory diseases in Jordan. Poult. Sci., 87(3): 444-448.

23. Pantin-Jackwood, M.J., Costa-Hurtado, M., Miller, P.J., Afonso, C.L., Spackman, E., Kapczynski, D.R., Shepherd, E., Smith, D. and Swayne, D.E. (2015) Experimental co-infections of domestic ducks with a virulent Newcastle disease virus and low or highly pathogenic avian influenza viruses. Vet. Microbiol., 177(1-2): 7-17.

24. Costa-Hurtado, M., Afonso, C.L., Miller, P.J., Shepherd, E., Cha, R.M., Smith, D., Spackman, E., Kapczynski, D.R., Suarez, D.L., Swayne, D.E. and Pantin-Jackwood, M.J. (2015) Previous infection with virulent strains of Newcastle disease virus reduces highly pathogenic avian influenza virus replication, disease, and mortality in chickens. Vet. Res., 46(1): 97.

25. Dortmans, J.C.F., Rottier, P.J.M., Koch, G. and
Peeters, B.P.H. (2010) The viral replication complex is associated with the virulence of Newcastle disease virus. J. Virol., 84(19): 10113-10120.

26. Liu, W., Ding, W., Kong, J., Wang, H., Hu, S., He, H., Zhang, R. and Liu, X. (2003) The interference in the virus propagation in chicken embryo and in the HI test between the Newcastle disease virus and the H9 subtype influenza virus. Chin. Poult. Sci., 7(3): 1-6.

27. Bonfante, F., Cattoli, G., Leardini, S., Salomoni, A., Mazzetto, E., Davidson, I., Haddas, R. and Terregino, C. (2017) Synergy or interference of an H9N2 avian influenza virus with a velogenic Newcastle disease virus in chickens is dose-dependent. Avian Pathol., 46(5): 488-496.

28. Dianzani, F. (1975) Viral interference and interferon. Ric. Clin. Lab., 5(3): 196-213.

29. Murphy, F.A., Gibbs, E.P.J., Horzinek, M.C. and Studdert, M.J. (1999) Veterinary Virology. $3^{\text {rd }}$ ed. Academic Press, San Diego.

30. Ferreira, L., Villar, E. and Muñoz-Barroso, I. (2004) Gangliosides and N-glycoproteins function as Newcastle disease virus receptors. Int. J. Biochem. Cell Biol., 36(11): 2344-2356.

31. Sonnenfeld, G. and Merigan, T.C. (1979) The role of interferon in viral infections. Springer Semin. Immunopathol., 2(3): 311-338. 